\title{
Conciliação Medicamentosa em Cuidados Paliativos Oncológicos
}

doi: https://doi.org/10.32635/2176-9745.RBC.2021v67n4.1360

\author{
Medication Reconciliation in Oncologic Palliative Care \\ Conciliación de la Medicación en Cuidados Paliativos Oncológicos
}

Maria Carolina Peçanha Fernandes; Luciana Favoreto Vieira Mattos²; Maria Fernanda Barbosa³

RESUMO

Introduçáo: A ausência de informações a respeito dos medicamentos utilizados pelos pacientes pode causar erros de medicaçôes. Assim, a comunicação entre profissionais de saúde, pacientes e familiares é primordial para a segurança do paciente nos diferentes níveis de atençáo à saúde. Os farmacêuticos clínicos podem realizar a conciliaçáo de medicamentos e atuar em colaboração com outros profissionais, objetivando otimizar a farmacoterapia e melhorar a segurança do paciente. As pessoas sob Cuidados Paliativos costumam fazer uso de polifarmácia e, quando não acompanhadas pelos profissionais de saúde, estão susceptíveis a potenciais discrepâncias não intencionais causadas por comunicaçáo inadequada. Objetivo: Analisar o perfil das conciliaçóes medicamentosas em pacientes que estão sob Cuidados Paliativos Oncológicos. Método: Estudo transversal, analítico e descritivo. Foram analisadas todas as visitas de conciliaçóes realizadas na admissáo dos pacientes, na unidade IV do Instituto Nacional de Câncer José Alencar Gomes da Silva (HCIV/INCA), no período de junho a novembro de 2018. Resultados: Realizaram-se 194 visitas, nas quais foram identificadas 1.770 discrepâncias (78,2\%), sendo 93,8\% intencionais, $0,7 \%$ intencionais documentadas e 5,4\% não intencionais. Todas as prescrições apresentaram pelo menos uma discrepância e 34,6\% foram totalmente modificadas pelo prescritor no ato da admissão. Foram realizadas 112 intervenções farmacêuticas relacionadas à conciliação medicamentosa. Conclusão: As principais discrepâncias encontradas, inclusão de medicamentos e ajustes de dose ressaltam a importância da presença de farmacêuticos clínicos no momento da admissão do paciente, em que foi possível ajustar a farmacoterapia, em conjunto com corpo clínico, contribuindo para a melhoria do perfil de prescrição.

Palavras-chave: Reconciliação de Medicamentos; Cuidados Paliativos; Institutos de Câncer; Segurança do Paciente; Brasil.

\section{ABSTRACT}

Introduction: The lack of information about the medications used by the patient can cause medication errors, so communication between health professionals, patients and family members is paramount for patient safety at different levels of attention to health. Clinical pharmacists can perform drug reconciliation and work in collaboration with other professionals to optimize pharmacotherapy and improve the patient's safety. Patients in Palliative Care tend to use polypharmacy, and when not accompanied by health professionals are susceptible to potential unintentional discrepancies caused by poor communication. Objective: To analyze the characteristics of the profile of drug reconciliations in patients who are under Oncologic Palliative Care. Method: Cross-sectional, analytical, and descriptive study. All the reconciliation visits performed at the admission of the patients were analyzed in the hospitalization unit of the National Cancer Institute José Alencar Gomes da Silva (HCIV/INCA), from June to November 2018. Results: A total of 194 visits were conducted, where 1,770 discrepancies (78.2\%) were found, $93.8 \%$ intentional, $0.7 \%$ intentional documented and $5.4 \%$ unintentional. All the prescriptions presented at least one discrepancy and $34.5 \%$ were totally modified by the prescriber on admission. There were 112 pharmaceutical interventions related to medication reconciliation. Conclusion: The main discrepancies found, inclusion of drugs and dose adjustments, highlights the importance of the presence of clinical pharmacists at the time of the patient's admission, when it was possible to adjust pharmacotherapy, together with the clinical staff and contributing to the improvement of the prescription profile.

Key words: Medication Reconciliation; Palliative Care; Cancer Care Facilities; Patient Safety; Brazil.

\section{RESUMEN}

Introducción: La falta de información sobre los medicamentos utilizados por el paciente puede generar errores de medicación, por lo que la comunicación entre los profesionales de la salud, los pacientes y los familiares es fundamental para la seguridad del paciente en los diferentes niveles de atención. Los farmacéuticos clínicos pueden realizar la conciliación de fármacos y trabajar en colaboración con otros profesionales para optimizar la farmacoterapia y mejorar la seguridad del paciente. Las personas que reciben Cuidados Paliativos suelen utilizar la polifarmacia y, cuando no están acompańadas de profesionales de la salud, son susceptibles a posibles discrepancias no intencionadas provocadas por una comunicación inadecuada. Objetivo: Analizar el perfil de las conciliaciones de fármacos en pacientes que se encuentran en Cuidados Oncológicos Paliativos. Método: Estudio transversal, analítico y descriptivo. Se analizaron todas las visitas de conciliación realizadas al ingreso de pacientes en la unidad de internación del Instituto Nacional del Cáncer José Alencar Gomes da Silva (HCIV/ INCA), de junio a noviembre de 2018. Resultados: Se realizaron 194 visitas, durante las cuales Se identificaron 1.770 discrepancias $(78,2 \%)$, de las cuales $93,8 \%$ fueron intencionales, $0,7 \%$ fueron documentadas y $5,4 \%$ fueron no intencionales. Todas las prescripciones mostraron al menos una discrepancia y el $34,5 \%$ se modificó por completo por el prescriptor al ingreso. Se realizaron 112 intervenciones farmacéuticas relacionadas con la conciliación de fármacos. Conclusión: Las principales discrepancias encontradas, inclusión de medicamentos y ajustes de dosis, resaltan la importancia de la presencia de farmacéuticos clínicos en el momento del ingreso del paciente, donde fue posible ajustar la farmacoterapia, junto con el personal clínico y contribuyendo a la mejora clínica de la prescripción. Palabras claves: Conciliación de Medicamentos; Cuidados Paliativos; Instituciones Oncológicas; Seguridad del Paciente; Brasil.

\footnotetext{
1-3Instituto Nacional de Câncer José Alencar Gomes da Silva (INCA), Unidade de Cuidados Paliativos. Rio de Janeiro (RJ), Brasil. E-mails: carolina.pecanha@hotmail. com; luciana.mattos@inca.gov.br; mbarbosa@inca.gov.br. Orcid iD: https://orcid.org/0000-0002-8899-1421; Orcid iD: https://orcid.org/0000-0003-2772-9697; Orcid iD: https://orcid.org/0000-0002-2018-6151

Endereço para correspondência: Maria Carolina Peçanha Fernandes. Rua da Passagem, 7, apto. 1204 - Botafogo. Rio de Janeiro (RJ), Brasil. CEP $22290-030$. E-mail: carolina.pecanha@hotmail.com
} 


\section{INTRODUÇÃO}

Sabe-se que aproximadamente $60 \%$ dos erros de medicação ocorrem durante a transição de nível de assistência à saúde, mais especificamente em prescriçôes médicas feitas na internação ou na alta hospitalar. A conciliação de medicamentos é um serviço realizado pelo farmacêutico, em que há a comparação dos medicamentos utilizados previamente e dos medicamentos prescritos. Tem grande importância clínica para os pacientes, uma vez que aumenta a segurança por promover reduçáo considerável de potenciais erros de medicação e diminuir as divergências encontradas entre os medicamentos já utilizados e as prescrições hospitalares realizadas no ato da internação ${ }^{1}$.

A The Joint Commission (TJC) entende que a conciliação pode reduzir erros e eventos adversos relacionados a medicamentos por evitar omissóes, duplicidades e erros de dose $\mathrm{d}^{2,3}$. Por esse motivo, a TJC propôs, em 2003, pela primeira vez, incluir a conciliação medicamentosa nos parâmetros de qualidade avaliados com o objetivo principal de melhorar a segurança do paciente. Em 2006, todas as organizaçôes de saúde credenciadas por esse órgão tiveram que desenvolver procedimentos de conciliação medicamentosa ${ }^{4}$.

Os principais erros de medicação que ocorrem nos pontos de transição do paciente, pelos níveis assistenciais de saúde, relacionam-se a: (i) informaçôes incorretas ou incompletas dos medicamentos utilizados, como: omissáo de dose, duplicidade terapêutica; (ii) interaçóes medicamentosas; e (iii) erros quanto à dose, à posologia, a medicamentos não justificados e à via de administração ${ }^{5,6}$. No contexto do paciente oncológico, prescrever os medicamentos, evitando a polifarmácia e as interaçóes medicamentosas, é a grande dificuldade. Essa dificuldade é ainda mais evidente no âmbito dos Cuidados Paliativos, em que os pacientes requerem o controle de sete a dez sintomas, sendo a dor o mais comum deles ${ }^{7-9}$. Sendo assim, este estudo teve por objetivo analisar o perfil da conciliaçáo de medicamentos em pacientes que estâo sob Cuidados Paliativos Oncológicos, em um hospital de referência do Sistema Único de Saúde.

\section{MÉTODO}

Estudo transversal, analítico e descritivo, realizado de junho a novembro de 2018, com pacientes em Cuidados Paliativos no Hospital do Câncer IV do Instituto Nacional de Câncer José Alencar Gomes da Silva (HCIV/INCA), cuja conciliação de medicamentos já era uma rotina do Serviço de Farmácia.

O Serviço da Farmácia Clínica é ofertado em 28 leitos da unidade de Cuidados Paliativos do INCA.
Diariamente, os pacientes são visitados na admissão da internação pela farmacêutica responsável nas primeiras 24 horas de internação. Os pacientes admitidos em feriados ou finais de semana são visitados no primeiro dia útil seguinte. Foram incluídos no estudo todos os pacientes que internaram nesses leitos no referido período, totalizando 194 pacientes. Pacientes menores de 18 anos, que não utilizavam nenhum medicamento regularmente, que estavam sem acompanhante e/ou com impossibilidade de se comunicar verbalmente, foram excluídos do estudo.

Durante o processo de conciliação, as novas prescriçôes foram acompanhadas e comparadas com a lista de medicamentos que o paciente utilizava anteriormente. Quando necessário, as evoluçōes descritas em prontuários foram analisadas. Após a avaliação dos dados, foram realizadas intervençóes junto aos médicos, quando encontradas discrepâncias não justificadas. Os dados foram coletados por meio de entrevista com os pacientes e/ou acompanhantes, bem como nos prontuários físicos e eletrônicos. $\mathrm{O}$ formulário preenchido pelo farmacêutico apresentava as seguintes variáveis relacionadas ao paciente: idade, diagnósticos, sintomas e Pontuação de Desempenho de Karnofsky (KPS $)^{10}$, que classifica a funcionalidade do paciente (quanto menor, menos funçóes consegue desempenhar). Quanto ao tratamento instituído ao paciente, foram avaliados: (a) medicamentos prescritos, nome do fármaco na denominação comum brasileira (DCB), dose, via de administraçáo e disponibilidade na instituição; (b) se os medicamentos em uso pelo paciente antes da internação constavam nas prescriçôes durante a internação hospitalar; (c) se existiam justificativas nas alteraçôes dos medicamentos; (d) aceitação pelo corpo clínico das intervenções farmacêuticas.

Toda diferença observada entre a lista de medicamentos, que o paciente fazia uso antes da internação, e a prescrição hospitalar vigente foi classificada como discrepância; nesta, se incluem alteraçôes de via de administração, forma farmacêutica, dose ou posologia e inclusão, exclusão ou substituiçáo de medicamento. Foram consideradas discrepâncias intencionais aquelas em que houve decisão médica justificada quanto à alteração dos medicamentos. Além disso, quando estas tinham registro em prontuário eletrônico, ou físico, foram classificadas como discrepâncias intencionais documentadas. Discrepâncias não intencionais foram aquelas em que ocorreram omissão de medicamento prescrito anteriormente, alteração na dose, frequência ou via de administraçáo diferente de um medicamento já utilizado pelo paciente, sem justificativa, bem como terapia duplicada ${ }^{11,12}$. Nesses casos, avaliaram-se as intervençóes realizadas e se estas foram ou náo aceitas. As classes terapêuticas dos medicamentos envolvidos 
nas discrepâncias foram categorizadas de acordo com a Classificaçáo Anatomical Therapeutic Chemical (ATC) ${ }^{13}$.

Os dados foram transcritos para uma planilha eletrônica e analisados com auxílio do software Microsoft Office Excel ${ }^{\circ}$.

Esta pesquisa foi aprovada pelo Comitê de Ética em Pesquisa do INCA, sob o número de parecer 2683715 e CAAE: 89429118.5.0000.5274, e desenvolvida em consonância com as Diretrizes preconizadas pelas Resoluções números 466/2012 14 e 510/2016 ${ }^{15}$ do Conselho Nacional de Saúde.

\section{RESULTADOS}

Foram realizadas 194 visitas de conciliação de junho a novembro de 2018. A maioria dos participantes era do sexo feminino (58,3\%), com mediana de idade 60 e o KPS com mediana de 40, o que significa que o paciente está incapaz, requer cuidados e assistência médica frequente. O câncer de abdômen foi o mais frequente (27,3\%), seguido dos cânceres de cabeça e pescoço $(16,0 \%)$ e ginecológico $(17,5 \%)$. Sessenta e quatro pacientes $(33 \%)$ foram encaminhados do HCI, HCII, HCIII e $130(67,1)$ já estavam no HCIV, sendo acompanhados pelo Serviço de Assistência Domiciliar (AD) ou pelo Ambulatório (AMB).

Os motivos de internaçáo foram agrupados em oito categorias, sendo que um paciente poderia apresentar mais de um motivo. A maior causa de internação foi o controle de sintomas, totalizando 165 (67\%) ocorrências. Os sintomas mais relatados foram dor, vômito, dispneia, confusão e/ou desorientação e fadiga. Trinta pacientes $(11,1 \%)$ foram admitidos na internação por transferência direta das outras unidades hospitalares do INCA; $23(8,5 \%)$ internaram por alteração nos parâmetros bioquímicos; $14(5,2 \%)$ por problemas com óstios, naqueles portadores de sonda nasogástrica, traqueostomia, gastrostomia, ileostomia e/ou, jejunostomia; oito $(3,0 \%)$ para a realização de procedimentos; e seis $(2,2 \%)$ por fragilidade na rede de apoio para os cuidados (Tabela 1).

Na Figura 1, observam-se os 1.616 medicamentos das prescriçôes anteriores às internaçôes dos participantes, agrupados por classe medicamentosa de acordo com ATC. A mediana de medicamentos antes da internaçáo era de oito, com maior prevalência de analgésicos $(21,5 \%)$, seguido de antieméticos $(13,4 \%)$, laxativos $(10,6 \%)$, protetor gástrico $(9,4 \%)$, corticoides $(7,6 \%)$, antiepilépticos (6,6\%), anti-hipertensivos (6,5\%). Os demais medicamentos, pertencentes a 29 classes diferentes, foram classificados como outros $(24,4 \%)$.

Do total de 194 prescrições, apenas 41 (21,1\%) tiveram menos de $50 \%$ dos medicamentos modificados e $67(34,6 \%) 100 \%$ da receita anterior modificada
Tabela 1. Características dos 194 participantes visitados no período de junho a novembro de 2018

\begin{tabular}{|c|c|c|}
\hline Sexo & $N(=194)$ & $\%$ \\
\hline Feminino & 113 & 58,3 \\
\hline Masculino & 81 & 41,7 \\
\hline \multirow[t]{2}{*}{ Idade } & Mediana & Variação \\
\hline & 60 & $21-89$ \\
\hline \multirow[t]{2}{*}{ KPS } & Mediana & Variaçãa \\
\hline & 40 & $10-80$ \\
\hline Tipo de câncer primário & $N(=194)$ & $\%$ \\
\hline Abdômen & 53 & 27,3 \\
\hline Ginecológico & 34 & 17,5 \\
\hline Cabeça e pescoço & 31 & 16,0 \\
\hline Mama & 27 & 13,9 \\
\hline Pulmão & 19 & 9,8 \\
\hline Pele & 9 & 4,6 \\
\hline TOC & 7 & 3,6 \\
\hline SNC & 5 & 2,6 \\
\hline Próstata & 5 & 2,6 \\
\hline Indefinido & 3 & 1,5 \\
\hline Pênis & 1 & 0,5 \\
\hline Origem da prescriçãa & $N(=194)$ & $\%$ \\
\hline HCIV AMB & 75 & 38,7 \\
\hline HCIV AD & 55 & 28,3 \\
\hline $\mathrm{HCl}$ & 37 & 19,1 \\
\hline $\mathrm{HCll}$ & 16 & 8,2 \\
\hline $\mathrm{HCIII}$ & 11 & 5,7 \\
\hline Motivos das internações & $N(=483)$ & $\%$ \\
\hline Controle de sintomas & 165 & 61,1 \\
\hline $\begin{array}{l}\text { Parâmetros bioquímicos } \\
\text { alterados }\end{array}$ & 23 & 8,5 \\
\hline $\begin{array}{l}\text { Transferências entre } \\
\text { unidades }\end{array}$ & 30 & 11,1 \\
\hline $\begin{array}{l}\text { Problemas com óstios } \\
\text { (sondas, GTT, TQ) }\end{array}$ & 14 & 5,2 \\
\hline Queda do estado geral & 13 & 4,8 \\
\hline Desidratação & 11 & 4,1 \\
\hline Exame/procedimento & 8 & 3,0 \\
\hline $\begin{array}{l}\text { Fragilidade na rede de } \\
\text { apoio }\end{array}$ & 6 & 2,2 \\
\hline
\end{tabular}

Legenda: KPS = Pontuaçáo de desempenho de Karnofsky; TOC = Tumor ósseo e conectivo; $\mathrm{SNC}=$ Sistema nervoso central; $\mathrm{HCI}=$ Hospital do Câncer I; HCII = Hospital do Câncer II, HCIII = Hospital do Câncer III; HCIV = Hospital do Câncer IV; $\mathrm{AMB}=$ Ambulatório; $\mathrm{AD}=$ Assistência domiciliar; $\mathrm{GTT}$ = gastrostomia; $\mathrm{TQ}=$ Traqueostomia. 


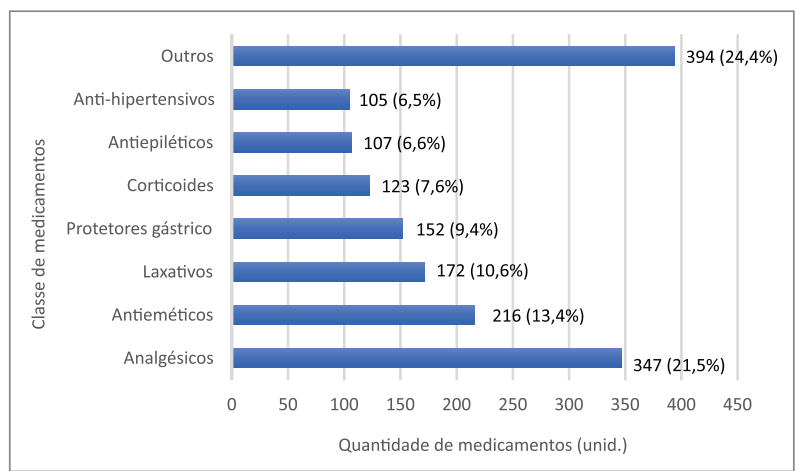

Figura 1. Classe dos medicamentos, segundo a ATC, utilizados pelos pacientes antes da conciliação medicamentosa

pelo prescritor no ato da admissão (Tabela 2). Foram identificadas 1.770 discrepâncias, nas quais 592 (33,4\%) medicamentos tiveram alteração na via de administração, $429(24,2 \%)$ foram omitidos, 389 (22,0\%) sofreram mudança na dose, $346(19,6 \%)$ discrepâncias na frequência e $14(0,8 \%)$ duplicidades.

Quanto às classificaçôes, 1.661 (93,8\%) foram intencionais, $13(0,8 \%)$ destas foram intencionais documentadas e 96 (5,4\%) foram discrepâncias não intencionais. No total, foram realizadas 112 intervençôes de conciliação e todas foram conciliadas, entretanto 16 não foram aceitas e, portanto, classificadas como discrepâncias intencionais. A partir das intervençôes aceitas (96), 41 (42,7\%) medicamentos omitidos foram incluídos, $30(31,3 \%)$ tiveram ajuste de posologia, 18 $(18,1 \%)$ foram excluídos e cinco $(5,2 \%)$ medicamentos foram substituídos por outro da mesma classe terapêutica, por desabastecimento.

\section{DISCUSSÃO}

Em todas as transiçôes de cuidados, os pacientes devem ser assistidos para garantir a segurança no uso de medicamentos: durante a admissão, com a coleta de informaçóes precisas sobre os medicamentos previamente utilizados, durante as transiçóes ao longo da internação, e posteriormente na alta hospitalar, visando à prevenção de erros de automedicaçáo. Nessa perspectiva, o processo de conciliação medicamentosa contribui para diminuir erros de prescrição, minimizando os riscos para os doentes, em especial aqueles com câncer ${ }^{16}$.

Neste estudo, observa-se que os pacientes sob Cuidados Paliativos Oncológicos utilizam, predominantemente, medicamentos para controle de sintomas, justificados pela própria doença ou pelos efeitos adversos causados pelos medicamentos. Um exemplo disso são os analgésicos opioides, que têm como efeito adverso comum a constipação, e consequentemente a prescrição de laxativos ${ }^{17-19}$. Percebe-se que a dor foi o sintoma mais
Tabela 2. Características dos resultados das conciliações farmacêuticas realizadas no período de junho a novembro de 2018

\begin{tabular}{|c|c|c|}
\hline $\begin{array}{l}\text { Medicamentos } \\
\text { alterados por } \\
\text { prescrição }\end{array}$ & $N(=194)$ & $\%$ \\
\hline$<50 \%$ & 41 & 21,1 \\
\hline$>50 \%$ a $90 \%$ & 86 & 44,3 \\
\hline $100 \%$ & 67 & 34,6 \\
\hline Tipos de discrepâncias & $N(=1.770)$ & $\%$ \\
\hline Via de administração & 592 & 33,4 \\
\hline Omissão & 429 & 24,2 \\
\hline Dose & 389 & 22,0 \\
\hline Frequência & 346 & 19,6 \\
\hline Duplicidade & 14 & 0,8 \\
\hline $\begin{array}{l}\text { Classificação das } \\
\text { discrepâncias }\end{array}$ & $N(=1.770)$ & $\%$ \\
\hline Discrepâncias intencionais & 1661 & 93,8 \\
\hline $\begin{array}{l}\text { Discrepâncias intencionais } \\
\text { documentadas }\end{array}$ & 13 & 0,8 \\
\hline $\begin{array}{l}\text { Discrepâncias não } \\
\text { intencionais }\end{array}$ & 96 & 5,4 \\
\hline $\begin{array}{l}\text { Tipos das intervenções } \\
\text { aceitas }\end{array}$ & $\mathbf{N}(=96)$ & $\%$ \\
\hline Inclusão de medicamento & 41 & 42,7 \\
\hline $\begin{array}{l}\text { Ajuste de dose ou } \\
\text { posologia }\end{array}$ & 30 & 31,3 \\
\hline Exclusão de medicamento & 18 & 18,1 \\
\hline $\begin{array}{l}\text { Substituição por } \\
\text { medicamento da mesma } \\
\text { classe terapêutica }\end{array}$ & 5 & 5,2 \\
\hline $\begin{array}{l}\text { Alteração da forma } \\
\text { farmacêutica }\end{array}$ & 1 & 1,0 \\
\hline $\begin{array}{l}\text { Alteração da via de } \\
\text { administração }\end{array}$ & 1 & 1,0 \\
\hline
\end{tabular}

relatado em pacientes oncológicos que estâo sob Cuidados Paliativos ${ }^{20-22}$, diferente de outros trabalhos publicados que possuem a maioria dos pacientes utilizando medicamentos de doenças crônicas não transmissíveis ${ }^{23-25}$, por possuírem populaçôes estudadas diferentes.

Os resultados das classificaçóes das discrepâncias se assemelham ao estudo realizado por Lindenmeyer et al. ${ }^{26}$, em um hospital público da Regiāo Sul do Brasil, onde, na admissão hospitalar de pacientes onco-hematológicos, a maior parte das discrepâncias foi identificada como intencional. Esses achados foram justificados pela condiçấo clínica atual dos pacientes, alteraçôes de doses, frequência ou via de administração, e pela substituição por outros medicamentos padronizados na instituição $0^{26}$.

Como mostrado anteriormente, $0,7 \%$ das discrepâncias foram documentadas; ou seja, estavam 
descritas em prontuários, indicando que o número exacerbado de discrepâncias não documentadas pode estar diretamente relacionado às discrepâncias náo intencionais pela falta de registro ou da sua qualidade sobre os medicamentos utilizados pelo paciente. Essas informações são fundamentais para garantir sua segurança e a farmacoterapia adequada ${ }^{27,28}$.

Os tipos de discrepâncias mais prevalentes neste estudo - via de administração e omissão de medicamentos - podem ser justificados por uma particularidade dos cuidados de fim de vida, em que há priorização do conforto. Para isso, são realizados a avaliação holística do sofrimento do paciente e o uso da via mais adequada para o manejo dos sintomas e melhora da qualidade de $\operatorname{vida}^{29}$. Assim, intervençóes, como as que visam à troca de via de administração, podem ser exemplificadas como a alteração da via oral para via intravenosa, uma vez que o paciente não consiga ingerir comprimidos. A discrepância "omissão de medicamentos" viabiliza a intervenção farmacêutica "inclusão de medicamentos". Ao analisar o motivo dessas intervençóes, verifica-se que, do total, $17 \%$ eram referentes a medicamentos indisponíveis para dispensação no hospital, e foram trazidos de casa pelos pacientes e seus cuidadores; $36,6 \%$ eram medicamentos que os pacientes faziam uso em razão da sua comorbidade, como medicamentos laxativos, analgésicos e antieméticos; e os demais eram medicamentos de uso crônico como anti-hipertensivos e medicamentos de controle especial, como ansiolíticos, antidepressivos e antipissicóticos. Além disso, houve um índice elevado na intervenção de alteração na posologia ou dose; neste caso, verifica-se que $46 \%$ foram em medicamentos analgésicos e $30 \%$ em antieméticos. O que reforça a característica da população estudada, uma população polissintomática.

Segundo Herledan et al. ${ }^{16}$, uma revisão sistemática que avaliou o impacto clínico e econômico da conciliaçáo medicamentosa mostrou que há impacto significativo quando se trata de erros de medicação detectados com a conciliação, e esse benefício pôde ser observado em ambos os ambientes (ambulatoriais e internaçôes), seja na admissão ou alta. Os estudos selecionados nessa revisão também mostraram que a conciliação de medicamentos permite ao farmacêutico clínico realizar intervenções adicionais. Como mostra o presente trabalho, outras intervençôes além da conciliação foram aceitas, indicando a importância do olhar do farmacêutico na rotina clínica ${ }^{16}$.

Estudos recentes encontraram resistência por parte da equipe médica acarretando a baixa aceitaçáo das intervençôes, que foi justificada pelo reflexo de um serviço ainda em construção e do desconhecimento da açáo do profissional farmacêutico nesse âmbito, bem como da importância da conciliação para a segurança do paciente e o impacto econômico que essa prática pode gerar para o hospital ${ }^{30,31}$. Entretanto, neste trabalho, a participação do farmacêutico no processo se mostrou efetiva e com boa aceitaçáo, ao fazer com que o profissional se aproximasse da equipe de saúde, reforçando a importância da implantaçáo dessa rotina na instituição. No entanto, há que se considerar que este estudo envolveu apenas uma parte da unidade hospitalar, sendo sua principal limitação. Recursos humanos são necessários para implementar o processo de conciliação medicamentosa em toda a unidade. A implementação total marcará a ampliação do Serviço, o qual se tornará mais completo, uma vez que poderá incluir, além da conciliaçáo medicamentosa na alta, o acompanhamento farmacoterapêutico e a orientaçáo na alta.

Durante as primeiras 24 horas após a admissão, a entrevista realizada pelo farmacêutico, de forma detalhada, permitiu resgatar informaçóes importantes quanto ao uso de medicamentos e pôde contribuir para o controle de sintomas e o conforto do paciente. Mais estudos de conciliação de medicamentos em Cuidados Paliativos Oncológicos precisam ser realizados, a fim de incentivar a inserção do farmacêutico na prática clínica, além de contribuir para tratamento do doente.

\section{CONCLUSÃO}

As características dos pacientes em Cuidados Paliativos valorizam a prática da conciliação realizada por farmacêuticos, já que se trata de pacientes polissintomáticos, poliqueixosos e polimedicados. Esses pacientes apresentam estadiamento avançado da doença oncológica e, por isso, internam pela falta de controle de diversos sintomas, contudo, vale ressaltar que, além do câncer, muitas vezes são portadores de outras comorbidades como hipertensão, depressão e ansiedade. $\mathrm{O}$ perfil da conciliação não se distância da literatura e, com as informaçôes coletadas no momento da entrevista, o farmacêutico pode atuar no auxílio do manejo dos sintomas ao realizar outras intervençôes que não estão inseridas no processo de conciliação.

\section{CONTRIBUIÇÕES}

As autoras contribuíram substancialmente na concepção ou no planejamento do estudo; na obtenção, análise e/ou interpretação dos dados; na redação e revisão crítica; e aprovaram a versão final a ser publicada.

\section{AGRADECIMENTOS}

À Flávia C. Barcelos, Andréa A. Tofani e Renata R. V. Cataldo, farmacêuticas do INCA, pelo auxílio na interpretação dos dados, assim como na revisão crítica 
do estudo; à Prisilla B. Pujatti, amiga e orientadora do Aperfeiçoamento em radiofarmácia, pela ajuda, incentivo e apoio.

\section{DECLARAÇÃO DE CONFLITO DE INTERESSES}

Nada a declarar.

\section{FONTES DE FINANCIAMENTO}

Não há.

\section{REFERÊNCIAS}

1. Ketchum K, Grass CA, Padwojski A. Medication reconciliation: verifying medication orders and clarifying discrepancies should be standard practice. Am J Nurs. 2005;105(11):78-9, 81-2, 84-5. doi: https://doi. org/10.1097/00000446-200511000-00033

2. Rozich JD, Howard RJ, Justeson JM, et al. Standardization as a mechanism to improve safety in health care. Jt Comm J Qual Saf. 2004;30(1):5-14. doi: https://doi. org/10.1016/s1549-3741(04)30001-8

3. Joint Commission International. Joint Commission International accreditation standards for hospitals: including standards for Academic Medical Center Hospitals. 7th ed. Oakbrook Terrace, IL: JCI; 2021.

4. Delgado Sánchez O, Martínez López I, Crespí Monjo $\mathrm{M}$, et al. Conciliación de la medicación: asumamos la responsabilidad compartida. Farm. Hosp. 2008;32(2):634. doi: https://doi.org/10.1016/S1130-6343(08)72815-0

5. Gleason KM, McDaniel MR, Feinglass J, et al. Results of the Medications at Transitions and Clinical Handoffs (MATCH) study: an analysis of medication reconciliation errors and risk factors at hospital admission. J Gen Intern Med. 2010;25(5):441-7. doi: https://doi.org/10.1007/ s11606-010-1256-6

6. Conklin JR, Togami JC, Burnett A, et al. Care transitions service: a pharmacy-driven program for medication reconciliation through the continuum of care. Am J Health Syst Pharm. 2014;71(10):802-10. doi: https:// doi.org/10.2146/ajhp130589

7. Waller A, Caroline NL. Handbook of palliative care in cancer. 2nd. Oxford, UK: Butterworth-Heinemann; 2000.

8. Koh NY, Koo WH. Polypharmacy in palliative care: can it be reduced? Singapore Med J. 2002;43(6):279-83.

9. McNeil MJ, Kamal AH, Kutner JS, et al. The burden of polypharmacy in patients near the end of life. J Pain Symptom Manage. 2016;51(2):178-83. doi: https://doi. org/10.1016/j.jpainsymman.2015.09.003

10. Karnofsky DA, Abelmann WH, Craver LF, et al. The use of the nitrogen mustards in the palliative treatment of carcinoma. With particular reference to bronchogenic carcinoma. Cancer. 1948;1(4):634-56. doi: https:// doi.org/10.1002/1097-0142(194811)1:4<634::AIDCNCR2820010410>3.0.CO;2-L

11. World Health Organization. The high 5 s project: interim report. Geneva: WHO; 2013 Dec.

12. Brito AMG. Reconciliação e revisão da medicaçáo na unidade de hospitalização domiciliária [dissertação]. Almada, PT: Instituto Superior de Ciências da Saúde Egas Moniz; 2017.

13. ATC/DDD Index 2021 [Internet]. Oslo, NO: WHO Collaborating Centre for Drug Statistics Methodology. [date unknown] - [update 2020 Dec 17; cited 2018 Agu 30]. Available from: http://www.whocc.no/atcddd

14. Conselho Nacional de Saúde (BR). Resolução no 466, de 12 de dezembro de 2012. Aprova as diretrizes e normas regulamentadoras de pesquisas envolvendo seres humanos. Diário Oficial da União, Brasília, DF. 2013 jun 13; Seção 1:59.14.

15. Conselho Nacional de Saúde (BR). Resolução no 510, de 7 de abril de 2016. Dispóe sobre as normas aplicáveis a pesquisas em Ciências Humanas e Sociais cujos procedimentos metodológicos envolvam a utilização de dados diretamente obtidos com os participantes ou de informaçôes identificáveis ou que possam acarretar riscos maiores do que os existentes na vida cotidiana, na forma definida nesta Resolução. Diário Oficial da Uniāo. 2016 maio 24; Edição98, Seção I:44.15.

16. Herledan C, Baudouin A, Larbre V, et al. Clinical and economic impact of medication reconciliation in cancer patients: a systematic review. Support Care Cancer. 2020;28(8):3557-69. doi: https://doi.org/10.1007/ s00520-020-05400-5

17. Pappagallo M. Incidence, prevalence, and management of opioid bowel dysfunction. Am J Surg. 2001;182(5A Suppl):11S-18S. doi: https://doi.org/10.1016/s00029610(01)00782-6

18. De Luca A, Coupar IM. Insights into opioid action in the intestinal tract. Pharmacol Ther. 1996;69(2):103-15. doi: https://doi.org/10.1016/0163-7258(95)02053-5

19. Herndon CM, Jackson KC, Hallin PA. Management of opioid-induced gastrointestinal effects in patients receiving palliative care. Pharmacotherapy. 2002;22(2):240-50. doi: https://doi.org/10.1592/phco.22.3.240.33552

20. Mercadante S, Adile C, Caruselli A, et al. The palliativesupportive care unit in a comprehensive cancer center as crossroad for patients' oncological pathway. PloS One. 2016;11(6):e0157300. doi: https://doi.org/10.1371/ journal.pone. 0157300

21. Bruce CM, Smith J, Price AA. Study of the incidence and management of admissions for cancer-related symptoms in a district general hospital: the potential role of an acute oncology service. Clin Oncol (R Coll Radiol). 2017;29(9):e148-e155. doi: https://doi.org/10.1016/j. clon.2017.03.012 
22. Mercadante S, Marchetti P, Adile C, et al. Characteristics and care pathways of advanced cancer patients in a palliative-supportive care unit and an oncological ward. Support Care Cancer. 2018;26(6):1961-6. doi: https:// doi.org/10.1007/s00520-017-4037-5

23. Riechelmann RP, Tannock IF, Wang L, et al. Potential drug interactions and duplicate prescriptions among cancer patients. J Natl Cancer Inst. 2007;99(8):592-600. doi: https://doi.org/10.1093/jnci/djk130

24. Spalla LR, Castilho SR. Medication reconciliation as a strategy for preventing medication errors. Braz J Pharm Sci. 2016;52(1):143-50. doi: https://doi.org/10.1590/ S1984-82502016000100016

25. Santos FN. Avaliação do cuidado farmacêutico na conciliação de medicamentos em pacientes idosos com câncer [tese na Internet]. Ribeirão Preto, SP: Faculdade de Medicina de Ribeirão Preto; 2018. doi: https://doi. org/10.11606/T.17.2018.tde-23072018-135825

26. Lindenmeyer LP, Goulart VP, Hegele V. Reconciliação medicamentosa como estratégia para a segurança do paciente oncológico - resultados de um estudo piloto. Rev Bras Farm Hosp Serv Saúde São Paulo [Internet]. 2013 [acesso 2019 jan 11];4(4):51-5. Disponível em: http://www.v1.sbrafh.org.br/public/ artigos/2013040408000469BR.pdf

27. Alvim BA. Importância da implantação e desenvolvimento da reconciliação medicamentosa em hospitais [monografia]. Palmas, TO: Universidade Luterana do Brasil; 2015.

28. Vega TG, Sierra-Sánchez JF, Martínez-Bautista MJ, et al. Medication reconciliation in oncological patients: a randomized clinical trial. J Manag Care Spec Pharm. 2016;22(6):734-40. doi: https://doi.org/10.18553/ jmcp. 2016.15248

29. Visser C, Hadley G, Wee B. Reality of evidencebased practice in palliative care. Cancer Biol Med. 2015;12(3):193-200. doi: https://doi.org/10.7497/j. issn.2095-3941.2015.0041

30. Oliveira LDB. Análise econômica da conciliação de medicamentos em uma unidade de internação de um hospital de ensino [monografia]. Lagarto, SE: Universidade Federal de Sergipe; 2018.

31. Pimenta PA, Santos KFS, Silva CML, et al. Conciliação de medicamentos em um hospital de ensino de Sergipe: liçóes aprendidas na implementação de um serviço. Sci Plena. 2020;16(8):084501. doi: https://doi. org/10.14808/sci.plena.2020.084501 